Interdisciplinary Studies of Complex Systems

No. 19 (2021) 5-17

(C) Yu. Kondratiev

https://doi.org/10.31392/iscs.2021.19.005

UDC 517.937

\title{
Three infinities of Cusanus
}

\author{
Yuri Kondratiev ${ }^{1}$
}

\begin{abstract}
We consider a mathematical realization of the concept of bounded infinity introduced by Nicholas of Cusa about 600 years ago.

Keywords: types of infinities, combinatorics, Newton polynomials, correlation functions

2010 Mathematics Subject Classification: 60J25, 60J65, 60G22, 47A30
\end{abstract}

\section{Introduction}

Nicholas of Cusa (1401 - 11 August 1464), also referred to as Nicholas of Kues and Nicolaus Cusanus, was a German philosopher, theologian, jurist, mathematician and astronomer. One of the first German proponents of Renaissance humanism, he made spiritual and political contributions in European history. A notable example of this is his mystical or spiritual writings on "learned ignorance", as well as his participation in power struggles between Rome and the German states of the Holy Roman Empire.

As papal legate to Germany from 1446, he was appointed cardinal for his merits by Pope Nicholas V in 1448 and Prince-Bishop of Brixen two years later. In 1459, he became vicar general in the Papal States.

Nicholas has remained an influential figure. In 2001, the sixth centennial of his birth was celebrated on four continents and commemorated by publications on his life and work.

Most of Nicholas's mathematical ideas can be found in his essays, De Docta Ignorantia (Of Learned Ignorance), De Visione Dei (On the Vision of God) and On Conjectures. Actually, mathematical ideas of Nicholas were deeply related with his philosophy and, in certain sense, were consequences of philosophical structures. We will be concentrated on his concept of the infinity. Note that Nicholas considered infinities of different types. In the first case he is dealing with pure infinity. This object is characterized by coincidence of oppositorum (opposites). He argues that infinite set of natural numbers is possible if there exists an infinity, i.e., an infinite number. In fact, this number he understood as a limiting object. The concept of the limit was a pioneering idea of Nicholas that was much before Newton and Leibnitz. This infinity is absolute and invisible.

\footnotetext{
${ }^{1}$ BiBoS, University of Bielefeld, D-33615 Bielefeld, Germany; National Pedagogical Dragomanov University, Kyiv, Ukraine. kondrat@math.uni-bielefeld.de
} 
The second type of infinity is, in his terminology, the bounded infinity (BI). In this infinity we shall see separated elements which form such object. There shall be a transition from the absolute infinity to BI. Moreover, the intrinsic structure of BI is important. This infinity we call call the transfinite one. BI has a structure, even infinitely many of structures.

Finally, Nicholas introduced the third type of infinity. In this type any element of BI includes again the infinity as its intrinsic structure.

Our first aim is to give a rigorous mathematical meaning to the concept of BI. Secondly, because the infinity is related with a limit transition from the natural numbers, we are interesting to see how standard aspects of the theory of natural numbers will be extended to the limiting space of BI. And the last question we would like to discuss concern the third infinity. We will give a mathematical model of this object.

Definitely, to realize these aims we will need more extended point of view which will include possible relation with other disciplines where such concept is relevant. Interdisciplinary studies represent one of the main trends in modern science. Several essential problems in science and its applications require a combination and interaction of methods and ideas from different areas of our knowledge. However, there appear to be many practical difficulties in the realization of an interdisciplinary approach. One of the challenges is that subject matter specialists may only have a superficial familiarity with the research done in related fields outside of their core area of expertise. For example, we witness an active development of mathematical models in biology and ecology, but the usage of such models by the experts in these disciplines is restricted by the lack of mathematical skills. On the other hand, the models themselves may be too generic and high level to be of practical use. The only way to overcome these difficulties is to create a practical and patient collaboration between scientists from different disciplines.

Another traditional topic of discussions concerning interdisciplinary research is the relation between mathematics and natural sciences on one side and philosophy and social sciences on the other. In the time of Newton and Leibnitz the concept of Naturphilosophie was a commonly accepted basis for the unification of several scientific disciplines. Since then, the necessary specialization and inevitable dissipation of particular sciences resulted in the divergence of philosophy and natural sciences. Although concrete results in physics, biology, etc. are still very stimulating for the philosophical studies, we would like to show that there is a fruitful reverse influence. The aim of this work is to illustrate some natural applied aspects of particular philosophical concepts in a mathematical framework by choosing a concrete mathematical object for this illustration. Due to the interdisciplinary character of this journal, we restricted ourselves to a few basic observations on this subject. Consequently, our explanations are done at the less advanced mathematical level in order to be accessible by the non-mathematical audience. For the detailed mathematical descriptions of related structures we refer to [4].

The myth of Plato's Cave served as one of the motivations for developing his concept of a world of ideas and a world of things. In the dialogue "State" he gives several examples illustrating this concept. As we know, Plato considered mathematics as one of the most important building blocks used to construct 
his philosophical system. Mathematical theories can serve as simple and illustrative tools for the existence of a world of ideas and a world of things. In a number of model situations, we are dealing with objects that appear from our observations in physics, biology, ecology, etc., yet full understanding of the mathematical structures of these models requires consideration of more general mathematical theories, which under some canonical mapping leads to the model situations in question.

\section{From natural numbers to configurations}

In this section we will use partially our recent paper [5]. The set of natural numbers $\mathbb{N}=\{0,1,2, \ldots\}$ is a fundamental object in the mathematics. In certain sense $\mathbb{N}$ is the root of all modern mathematics. Other mathematical structures may be created as a logical development of this object. The latter motivated L. Kronecker who summarized "God made the integers, all else is the work of man". There is famous citation from I. Kant: "Two things fill the mind: the starry heavens above me and the moral law within me". a mathematician may continue: "and natural numbers given to my mind".

From the time of Pythagoras philosophers was trying to see hidden meaning of natural numbers and their mystical properties. Considering $\mathbb{N}$ as a set of real things in mathematics we will ask ourself about possible ideas behind these numbers. The myth of Plato's Cave served as one of the motivations for developing his concept of a world of ideas and a world of things. In the dialogue "State" he gives several examples illustrating this concept. As we know, Plato considered mathematics as one of the most important building blocks used to construct his philosophical system. Mathematical theories can serve as simple and illustrative tools for the existence of a world of ideas and a world of things. In a number of model situations, we are dealing with objects that appear from our observations in physics, biology, ecology, etc., yet full understanding of the mathematical structures of these models requires consideration of more general mathematical theories, which under some canonical mapping leads to the model situations in question.

The first and essentially obvious observation here is the following. a number $n \in \mathbb{N}$ we interpret as a number of objects (a population) located in a location space $X$. For simplicity we take $X=\mathbb{R}^{d}$. The collection of all $n$-point subsets (or configurations with $n$ elements) form a locally compact space $\Gamma^{(n)}\left(\mathbb{R}^{d}\right)$. It is the space (quite huge) of ideas for the number $n$. Then to $\mathbb{N}$ corresponds the set

$$
\Gamma_{0}\left(\mathbb{R}^{d}\right)=\cup_{n=0}^{\infty} \Gamma^{(n)}\left(\mathbb{R}^{d}\right)
$$

of all finite configurations.

We can consider additionally the set $\Gamma\left(\mathbb{R}^{d}\right)$ consisting all locally finite configurations. This set may be considered as the space of ideas which corresponds to the BI notion. Each configuration presents particular structure of BI. If we introduce a probability measure on $\Gamma\left(\mathbb{R}^{d}\right)$, then we can speak about random infinity.

Configuration spaces form an important and actively developing area in the infinite dimensional analysis. On one hand side, these spaces represent rich mathematical structures which combine, in a very non-trivial way, continuous 
and combinatoric aspects of the analysis. On the other hand side, configuration spaces give natural mathematical techniques for solving problems of mathematical physics, biology and ecology.

In such extension of $\mathbb{N}$ we arrive in the main question. Namely, most important mathematical theories related to natural numbers we need to develop to this new level. It concerns, first of all, the combinatorics that play central role in many mathematical structures and applications from probability theory to genetics. In this note we will try to show such possibility trying to be as much as possible on technically simple ground. To be friendly to more wide audience, we restrict out explanations to descriptions of main constructions and formulation of some particular results. For detailed discussions and extended references we refer to the recent paper [2].

\section{Classical combinatorics}

The combinatorics is dealing with the set of natural numbers $\mathbb{N}$ and relations between them. As an important object we introduce binomial coefficients:

$$
\left(\begin{array}{l}
n \\
k
\end{array}\right)=\frac{n(n-1) \ldots(n-k+1)}{k !}
$$

defined for $n \in \mathbb{N}$ and $0 \leq k \leq n$. Introducing the falling factorial $(n)_{k}$ we can write

$$
\left(\begin{array}{l}
n \\
k
\end{array}\right)=\frac{(n)_{k}}{k !}
$$

These coefficients may be extended using embedding $\mathbb{N} \subset \mathbb{R}$ to polynomials

$$
N_{k}(t):=\left(\begin{array}{l}
t \\
k
\end{array}\right)=\frac{t(t-1) \ldots(t-k+1)}{k !}=\frac{(t)_{k}}{k !}, t \in \mathbb{R}
$$

which are called Newton polynomials. For Newton polynomials hold ChuVandermond relations:

$$
(t+s)_{n}=\sum_{k=0}^{n}\left(\begin{array}{l}
n \\
k
\end{array}\right)(t)_{k}(s)_{n-k} .
$$

An alternative definition is given by the generation function

$$
e_{\lambda}(t):=e^{t \log (1+\lambda)}=\sum_{n=0}^{\infty} \frac{\lambda^{n}}{n !}(t)_{n}=\sum_{n=0}^{\infty} \lambda^{n} N_{n}(t) .
$$

Such transition to continuous variables makes possible to apply in discrete mathematics methods of analysis. Note that using many particular generation functions we may create different polynomial systems.

Transition to continuous variables makes possible to apply in discrete mathematics methods of analysis. In particular, let us define for functions $f: \mathbb{R} \rightarrow \mathbb{R}$ difference operators

$$
\left(D^{+} f\right)(t)=f(t+1)-f(t)
$$




$$
\left(D^{-} f\right)(t)=f(t-1)-f(t) .
$$

By a direct computation we obtain

$$
\begin{gathered}
D^{+}(t)_{n}=n(t)_{n-1}, \\
D^{-}(t)_{n}=-n(t-1)_{n-1} .
\end{gathered}
$$

Additionally,

$$
D^{+} e_{\lambda}(t)=\lambda e_{\lambda}(t)
$$

In this way we arrive in the framework of difference calculus closely related with the combinatorics [3]. There are specific questions inside of difference calculus as, e.g., an analysis of Newton series

$$
\sum_{n=0}^{\infty} a_{n} N_{n}(t)
$$

and many others.

For functions $a: \mathbb{N} \rightarrow \mathbb{R}$ we define $b: \mathbb{N} \rightarrow \mathbb{R}$ as

$$
b=K a, \quad b(n)=\sum_{k=0}^{n}\left(\begin{array}{l}
n \\
k
\end{array}\right) a(k) .
$$

This operator $K$ (aka combinatorial transform) is very useful in combinatorics and its inverse gives so-called inclusion-exclusion formula:

$$
a(n)=\sum_{k=0}^{n}\left(\begin{array}{l}
n \\
k
\end{array}\right)(-1)^{n-k} b(k) .
$$

Note that for $a: \mathbb{N} \rightarrow \mathbb{R}, a(j)=0, j \neq k, a(k)=1$

$$
(K a)(n)=\left(\begin{array}{l}
n \\
k
\end{array}\right)=k ! N_{k}(n) .
$$

\section{Spatial combinatorics}

Any $n \in \mathbb{N}$ we interpret as the size of a population. It is convenient in the study of population models. There is a natural generalization leading to spatial ecological models. Now we would like to consider objects located in a given locally compact space $X$. For simplicity we will work with the Euclidean space $\mathbb{R}^{d}$. For the substitution of $\mathbb{N}$ in this situation we can use two possible sets. Denote $\Gamma\left(\mathbb{R}^{d}\right)$ the set of all locally finite configurations (subsets) from $\mathbb{R}^{d}$ :

$$
\Gamma\left(\mathbb{R}^{d}\right)=\left\{\gamma \subset \mathbb{R}^{d}|| \gamma \cap K \mid<\infty \text {, any compact } K \subset \mathbb{R}^{d}\right\} .
$$

It is the first version of the space in the spatial (continuous) combinatoric we will use.

Another possibility, is to introduce the set of all finite configurations $\Gamma_{0}\left(\mathbb{R}^{d}\right)$. Then

$$
\Gamma_{0}\left(\mathbb{R}^{d}\right)=\cup_{n=0}^{\infty} \Gamma^{(n)}\left(\mathbb{R}^{d}\right),
$$


where $\Gamma^{(n)}\left(\mathbb{R}^{d}\right)$ denoted the set of all configurations with $n$ elements. We will see that in the continuous combinatoric the spaces $\Gamma\left(\mathbb{R}^{d}\right)$ and $\Gamma_{0}\left(\mathbb{R}^{d}\right)$ will play very different roles. It is a specific moment related with transition to the continuum. In this sense $\mathbb{N}$ is splitting in these two spaces of configurations that makes corresponding combinatorics essentially more reach and sophisticated.

Configuration spaces present beautiful combinations of discrete and continuous properties. In particular, in these spaces we have interesting differential geometry, differential operators and diffusion processes etc., see e.g. [1]. From the other hand side, discreteness of an individual configuration makes possible to introduce proper analog of the difference calculus.

Note from the beginning, that the analog of the extension $\mathbb{N} \subset \mathbb{R}$ now naturally play the pair $\Gamma\left(\mathbb{R}^{d}\right) \subset \mathcal{M}\left(\mathbb{R}^{d}\right)$ where we have in mind an imbedding of configurations in the space of discrete Radon measures on $\mathbb{R}^{d}$ and, as a result, in the space of all Radon mesures on $\mathbb{R}^{d}$ :

$$
\Gamma(X) \ni \gamma \mapsto \gamma(d x)=\sum_{M \times \mathbb{R}^{d} \in \gamma} \delta_{y}(d x) \in \mathcal{M}\left(\mathbb{R}^{d}\right) .
$$

Therefore, instead of pair

$$
\mathbb{N} \subset \mathbb{R}
$$

we have

$$
\Gamma\left(\mathbb{R}^{d}\right) \subset \mathcal{M}\left(\mathbb{R}^{d}\right) .
$$

As a result, the transition to "continuous" variables in the considered situation leads to functions on $\mathcal{M}\left(\mathbb{R}^{d}\right)$. In spatial combinatorics many objects will be measure-valued.

Now we will introduce an analog of the generation function from classical combinatorics. For a test function from the Schwarz space of test functions $\mathcal{D}\left(\mathbb{R}^{d}\right) 0 \leq \xi \in \mathcal{D}\left(\mathbb{R}^{d}\right)$ consider a function

$$
E_{\xi}(\omega)=e^{<\ln (1+\xi), \omega>}, \quad \omega \in \mathcal{D}^{\prime}\left(\mathbb{R}^{d}\right)
$$

that is a function on the space of Schwarz distributions. The power decomposition w.r.t. $\xi$ gives

$$
E_{\xi}(\omega)=\sum_{n=0}^{\infty} \frac{1}{n !}<\xi^{\otimes n},(\omega)_{n}>.
$$

Generalized kernels $(\omega)_{n} \in \mathcal{D}^{\prime}\left(\mathbb{R}^{n d}\right)$ are called infinite dimensional falling factorials on $\mathcal{D}^{\prime}\left(\mathbb{R}^{d}\right)$. Define binomial coefficients (Newton polynomials) on $\mathcal{D}^{\prime}\left(\mathbb{R}^{d}\right)$ as

$$
\left(\begin{array}{l}
\omega \\
n
\end{array}\right)=\frac{(\omega)_{n}}{n !}
$$

Note that these objects are defined now on the very big space of distributions. In particular cases we shall restrict them on the space of configuration or Radon measures.

In particular, infinite dimensional Chu-Vandermond relations on configurations is

$$
\left(\gamma_{1} \cup \gamma_{2}\right)_{n}=\sum_{k=0}^{n}\left(\begin{array}{l}
n \\
k
\end{array}\right)\left(\gamma_{1}\right)_{k} \otimes\left(\gamma_{2}\right)_{n-k} .
$$


Theorem 1. For $\omega \in \mathcal{M}\left(\mathbb{R}^{d}\right)$

$$
\begin{gathered}
(\omega)_{0}=1 \\
(\omega)_{1}=\omega \\
(\omega)_{n}\left(x_{1}, \ldots, x_{n}\right)=\omega\left(x_{1}\right)\left(\omega\left(x_{2}\right)-\delta_{x_{1}}\left(x_{2}\right)\right) \ldots\left(\omega\left(x_{n}\right)-\delta_{x_{1}}\left(x_{n}\right)-\ldots-\delta_{x_{n-1}}\left(x_{n}\right)\right) .
\end{gathered}
$$

In the particular case $\omega=\gamma=\left\{x_{i} \mid i \in \mathbb{N}\right\}$

$$
(\gamma)_{n}=n !\left(\begin{array}{l}
\gamma \\
n
\end{array}\right)=\sum_{\left\{i_{1} \ldots, i_{n}\right\} \subset \mathbb{N}} \delta_{x_{1}} \odot \ldots \odot \delta_{x_{n}},
$$

where $\delta_{x_{1}} \odot \ldots \odot \delta_{x_{n}}$ denotes symmetric tensor product.

We have

$$
\Gamma\left(\mathbb{R}^{d}\right) \ni \gamma \mapsto \gamma(d x) \in \mathcal{M}\left(\mathbb{R}^{d}\right) .
$$

Due to our construction

$$
(\gamma)_{n} \in \mathcal{M}\left(\mathbb{R}^{n d}\right)
$$

is a symmetric Radon masure. Therefore, we arrive in measure valued Newton polynomials. The latter is the main consequence of continuous combinatoric transition.

\section{Difference geometry for spatial combinatorics} ent)

For any $x \in \gamma$ define an elementary Markov death operator (death gradi-

$$
D_{x}^{-} F(\gamma)=F(\gamma \backslash x)-F(\gamma)
$$

and the tangent space $T_{\gamma}^{-}(\Gamma)=L^{2}\left(\mathbb{R}^{d}, \gamma\right)$. Then for $\psi \in C_{0}\left(\mathbb{R}^{d}\right)$

$$
D_{\psi}^{-} F(\gamma)=\sum_{x \in \gamma} \psi(x)(F(\gamma \backslash x)-F(\gamma))
$$

is the directional (difference) derivative.

Similarly, we define for $x \in \mathbb{R}^{d}$

$$
D_{x}^{+} F(\gamma)=F(\gamma \cup x)-F(\gamma)
$$

and the tangent space $T_{\gamma}^{-}(\Gamma)=L^{2}\left(\mathbb{R}^{d}, d x\right)$. Then for $\varphi \in C_{0}\left(\mathbb{R}^{d}\right)$

$$
D_{\varphi}^{+} F(\gamma)=\int_{\mathbb{R}^{d}} \varphi(x)(F(\gamma \cup x)-F(\gamma)) d x
$$

is another directional (difference) derivative.

For $\varphi \in C_{0}\left(\mathbb{R}^{d}\right)$ define a function

$$
E_{\varphi}(\gamma)=\exp (<\gamma, \log (1+\varphi)>), \quad \gamma \in \Gamma .
$$

It is the generation function for the system on falling factorials (Newton polynomials) on $\Gamma$ :

$$
E_{\varphi}(\gamma)=\sum_{n=0}^{\infty} \frac{1}{n !}<\varphi^{\otimes n},(\gamma)_{n}>
$$


Then

$$
D_{\psi}^{+} E_{\varphi}(\gamma)=<\varphi \psi>E_{\varphi}(\gamma)
$$

An explicit formula for the falling factorials (as measures on $\left(\mathbb{R}^{d}\right)^{n}$ ) is

$$
(\gamma)_{n}=\sum_{\left\{x_{1}, \ldots, x_{n}\right\} \subset \gamma} \delta_{x_{1}} \odot \delta_{x_{2}} \odot \cdots \odot \delta_{x_{n}},
$$

where $\delta_{x_{1}} \odot \delta_{x_{2}} \odot \cdots \odot \delta_{x_{n}}$ denotes the symmetric tensor product of measures.

The action of difference derivatives on Newton monomials is given by

$$
\begin{gathered}
D_{\psi}^{+}<\varphi^{(n)},(\gamma)_{n}>=n \int_{\mathbb{R}^{d}} \psi(x)<\varphi^{(n)}(x, \cdot),(\gamma)_{n-1}(\cdot)>d x, \\
D_{\psi}^{-}<\varphi^{(n)},(\gamma)_{n}>=-n \sum_{x \in \gamma} \psi(x)<\varphi^{(n)}(x, \cdot),(\gamma \backslash x)_{n-1}(\cdot)>.
\end{gathered}
$$

\section{Harmonic analysis on $\Gamma\left(\mathbb{R}^{d}\right)$}

Functions $G: \Gamma_{0}\left(\mathbb{R}^{d}\right) \rightarrow \mathbb{R}$ we call quasi-observables. Note that $G$ restricted on $\Gamma^{(n)}\left(\mathbb{R}^{d}\right)$ is given by a symmetric kernel $G^{(n)}\left(x_{1}, \ldots, x_{n}\right)$ and then

$$
G=\left(G^{(n)}\right)_{n=0}^{\infty}
$$

Functions $F: \Gamma\left(\mathbb{R}^{d}\right) \rightarrow \mathbb{R}$ we call observables. For a quasi-observable $G$ define an operator

$$
(K G)(\gamma)=\sum_{\eta \subset \gamma,|\eta|<\infty} G(\eta), \quad \gamma \in \Gamma\left(\mathbb{R}^{d}\right)
$$

that is an observable. To be well defined we need certain assumptions about $G[6]$.

For $G_{1}, G_{2}: \Gamma_{0}\left(\mathbb{R}^{d}\right) \rightarrow \mathbb{R}$ define

$$
\left(G_{1} \star G_{2}\right)(\eta)=\sum_{\eta_{1} \cup \eta_{2} \cup \eta_{3}=\eta} G_{1}\left(\eta_{1} \cup \eta_{2}\right) G_{2}\left(\eta_{2} \cup \eta_{3}\right) .
$$

Then

$$
K\left(G_{1} \star G_{2}\right)=K G_{1} K G_{2} .
$$

Let $\mu \in \mathcal{M}^{1}\left(\Gamma\left(\mathbb{R}^{d}\right)\right)$.

$$
\begin{gathered}
K: \operatorname{Fun}\left(\Gamma_{0}\right) \rightarrow \operatorname{Fun}(\Gamma) \\
K^{*}: \mathcal{M}^{1}(\Gamma) \rightarrow \mathcal{M}\left(\Gamma_{0}\right) . \\
K^{*} \mu=\rho, \quad \rho=\left(\rho^{(n)}\right)_{n=0}^{\infty} .
\end{gathered}
$$

The measure $\rho$ is called correlation measure for $\mu$ (Fourier transform of $\mu$ ).

Assume absolute continuity

$$
d \rho^{(n)}\left(x_{1}, \ldots, x_{n}\right)=\frac{1}{n !} k^{(n)}\left(x_{1}, \ldots, x_{n}\right) d x_{1} \ldots x_{n} .
$$


We call $k^{(n)}\left(x_{1}, \ldots, x_{n}\right), n \in \mathbb{N}$ correlation functions of the measure $\mu$.

Transition from measures to CFs is one of the main technical aspects of the analysis on CS in applications to dynamical problems.

Alternatively define the Bogoliubov functional

$$
B_{\mu}(\phi)=\int_{\Gamma\left(\mathbb{R}^{d}\right)} e^{<\gamma, \log (1+\phi>)} d \mu(\gamma)
$$

Assuming $B_{\mu}$ is holomorphic in $\phi \in L^{1}\left(\mathbb{R}^{d}\right)$ we obtain

$$
B_{\mu}(\phi)=\sum_{n=0}^{\infty} \frac{1}{n !} \int k^{(n)}\left(x_{1}, \ldots, x_{n}\right) \phi\left(x_{1}\right) \ldots \phi\left(x_{n}\right) d x_{1} \ldots d x_{n} .
$$

\section{From spatial to classical combinatorics}

Having developed combinatorial structures in the continuum, we may consider the inverse direction. Namely, how looks like our infinite-dimensional objects in the one dimensional reduction. Surprisingly, it may give some new structures even in this classical case.

Let $a, b: \mathbb{N} \rightarrow \mathbb{R}$. Define a convolution

$$
(a \star b)(n)=\sum_{j+k+l=n} a(j+k) b(k+l) .
$$

As before

$$
(K a)(n)=\sum_{k+0}^{n}\left(\begin{array}{l}
n \\
k
\end{array}\right) a(k)
$$

Then

$$
K(a \star b)=K a \cdot K b .
$$

Introduce coherent states

$$
\begin{gathered}
e_{\lambda}(\cdot): \mathbb{N} \rightarrow \mathbb{C}, e_{\lambda}(n)=\lambda^{n}, \quad \lambda \in \mathbb{C} . \\
\left(K e_{\lambda}\right)(n)=(1+\lambda)^{n} .
\end{gathered}
$$

The configuration space $\Gamma\left(\mathbb{R}^{d}\right)$ is the space of microscopic states in the classical statistical physics of continuous systems. A measure $\mu \in \mathcal{M}^{1}\left(\Gamma\left(\mathbb{R}^{d}\right)\right)$ is a macroscopic state of a continuous system in the statistical physics. Coming back we can interpret (a bit naively) a measure $\mu \in \mathcal{M}^{1}(\mathbb{N})$ as a state of $0 D$ system.

For example, the Poisson measure for $\sigma>0$ is defined as

$$
\pi_{\sigma}(n)=e^{-\sigma} \frac{\sigma^{n}}{n !}
$$

Several characteristics we can incorporate in such a case from the analysis on $\Gamma\left(\mathbb{R}^{d}\right)$. Introduce the Bogoliubov functional:

$$
B(\lambda)=\int_{\mathbb{R}_{+}}(1+\lambda)^{x} d \mu(x) .
$$




$$
(1+\lambda)^{x}=\sum_{n=0}^{\infty} \frac{\lambda^{n}}{n !}(x)_{n} .
$$

Theorem 2. Let $\mu \in \mathcal{M}^{1}\left(\mathbb{R}_{+}\right)$. Then $\mu(\mathbb{N})=1$ iff $B(\lambda)$ has a holomorphic extension.

Similarly we can define correlation measures

$$
\begin{gathered}
\int_{\mathbb{N}}(K a)(x) d \mu(x)=\int_{\mathbb{N}} a(x) d \rho_{\mu}(x) . \\
\rho_{\mu}(n)=\frac{1}{n !} \int_{\mathbb{N}}(x)_{n} d \mu(x)= \\
\sum_{m=n}^{\infty}\left(\begin{array}{l}
m \\
n
\end{array}\right) \mu(m) .
\end{gathered}
$$

\section{Third infinity}

\subsection{The Cone of Positive Discrete Measure-Valued Radon Measures}

We will denote $M$ the space of Radon measures $\mathcal{M}\left(\mathbb{R}^{d}\right)$ or $\Gamma\left(\mathbb{R}^{d}\right) \subset$ $\mathcal{M}\left(\mathbb{R}^{d}\right)$. The latter case will be of our main interest. Both spaces are Polish ones. Introduce a space $\mathcal{M}\left(\mathbb{R}^{d} \rightarrow M\right)$ of Radon measures on $\mathbb{R}^{d}$ with values in $M$. It means that for $\eta(d s, d x) \in \mathcal{M}\left(\mathbb{R}^{d} \rightarrow M\right)$ and $A$ bonded Borel subset of $\mathbb{R}^{d}$ we have $\eta(\cdot, A) \in M$.

Definition 3. 1) The cone of discrete measure-valued Radon measures is defined as follows:

$$
\mathbb{K} M\left(\mathbb{R}^{d}\right):=\left\{\eta=\sum_{i} \gamma_{i} \otimes \delta_{x_{i}} \in \mathcal{M}\left(\mathbb{R}^{d} \rightarrow M\right) \mid \gamma_{i} \in M, x_{i} \in \mathbb{R}^{d}\right\}
$$

By convention, the zero measure $\eta=0$ is included in $\mathbb{K} M\left(\mathbb{R}^{d}\right)$.

2) We denote the support of $\eta \in \mathbb{K} M\left(\mathbb{R}^{d}\right)$ by

$$
\tau(\eta):=\left\{x \in \mathbb{R}^{d} \mid 0 \neq \eta(\{x\})=: \gamma_{x}(\eta) \in M\right\} .
$$

If $\eta$ is fixed, we write $\gamma_{x}:=\gamma_{x}(\eta)$. We stress that $\tau(\eta)$ is not a configuration in general.

3) For $\eta, \xi \in \mathbb{K} M\left(\mathbb{R}^{d}\right)$ we write $\xi \subset \eta$ if $\tau(\xi) \subset \tau(\eta)$ and $\gamma_{x}(\xi)=\gamma_{x}(\eta)$ for all $x \in \tau(\xi)$. If additionally $|\tau(\xi)|<\infty$, we write $\xi \Subset \eta$.

4) For a function $f \in C_{c}\left(\mathbb{R}^{d}\right)$, denote the pairing with an element $\eta \in$ $\mathbb{K} M\left(\mathbb{R}^{d}\right)$ by

$$
\langle f, \eta\rangle:=\int_{\mathbb{R}^{d}} f(x) \eta(d s, d x)=\sum_{x \in \tau(\eta)} \gamma_{x} f(x)
$$

that is a measure on $\mathbb{R}^{d}$. 


\subsection{Plato's theory}

In the theory, Plato stated that observations in the real world are mere projections of higher forms or ideas. One way to picture this is the so-called cave allegory, which was recited by Ross (1951) as follows: "A company of men is imprisoned in an underground cave, with their heads fixed so that they can look only at the back wall of the cave. Behind them across the cave runs a wall behind which men pass, carrying all manner of vessels and statues which overtop the wall. Behind these again is a fire. The prisoners can only see the shadows $[\ldots]$ of the things carried behind the wall, and must take these to be the only realities" . Applied to our setting, the space $\mathbb{K} M\left(\mathbb{R}^{d}\right)$ is interpreted as the shadows projected onto the cave wall. On the other hand, the space $\Pi\left(M \times \mathbb{R}^{d}\right)$ which will be introduced below is the space of forms or ideas, represented by the objects carried in front of the fire. While the space $\mathbb{K} M\left(\mathbb{R}^{d}\right)$ is taken to be our reality, we use the space $\Pi\left(M \times \mathbb{R}^{d}\right)$ to define mathematical operations. The spaces are connected via the bijection $\mathcal{R}: \Pi\left(M \times \mathbb{R}^{d}\right) \rightarrow \mathbb{K} M\left(\mathbb{R}^{d}\right)$ introduced below. In accordance with the cave allegory, $\mathcal{R}$ is also called reflection mapping.

\subsection{Configuration Spaces}

As we will see in the next chapter, the Plato space $\Pi\left(M \times \mathbb{R}^{d}\right)$ is a specific subset of the so-called configuration space $\Gamma\left(M \times \mathbb{R}^{d}\right)$.

In general, the space of locally finite configurations $\Gamma\left(M \times \mathbb{R}^{d}\right)$ is the space of all subsets of $M \times \mathbb{R}^{d}$ which are finite in any bounded set $\Lambda$ in the metric space $M \times \mathbb{R}^{d}$. The following definition makes this notion more precise.

Definition 4. The space of locally finite configurations over $M \times \mathbb{R}^{d}$ is defined as

$$
\Gamma\left(M \times \mathbb{R}^{d}\right)=\left\{\gamma \subset M \times \mathbb{R}^{d}:|\gamma \cap \Lambda|<\infty \forall \Lambda \subset M \times \mathbb{R}^{d} \text { bounded }\right\}
$$

where $|\cdot|$ denotes the number of elements of a set.

The space $\Gamma\left(M \times \mathbb{R}^{d}\right)$ is exactly the third type infinity in the sense of Nicholas of Cusa. Namely, for each point $x \in \mathbb{R}^{d}$ from the discrete set that present bounded infinity we prescribed an internal characteristic $\gamma_{x}$ that is again an element of bounded infinity.

\subsection{Relation Between $\mathbb{K} M\left(\mathbb{R}^{d}\right)$ and $\Gamma\left(M \times \mathbb{R}^{d}\right)$}

In this section, we want to establish the connection between the configuration space $\Gamma\left(M \times \mathbb{R}^{d}\right)$ and the cone $\mathbb{K} M\left(\mathbb{R}^{d}\right)$. Our goal is to define a certain subspace $\Pi\left(M \times \mathbb{R}^{d}\right) \subset \Gamma\left(M \times \mathbb{R}^{d}\right)$ such that there exists a one-toone-correspondence between $\Pi\left(M \times \mathbb{R}^{d}\right)$ and $\mathbb{K} M\left(\mathbb{R}^{d}\right)$ in the following form:

$$
\mathcal{R}: \Pi\left(M \times \mathbb{R}^{d}\right) \rightarrow \mathbb{K} M\left(\mathbb{R}^{d}\right), \gamma=\sum_{(\mu, x) \in \gamma} \delta_{(\mu, x)} \mapsto \sum_{(\mu, x) \in \gamma} \mu \otimes \delta_{x}
$$

In terms of Plato's theory, this mapping takes ideas $\gamma \in \Pi\left(M \times \mathbb{R}^{d}\right)$ and projects (or reflects) them to objects $\eta \in \mathbb{K} M\left(\mathbb{R}^{d}\right)$. Obviously, $\mathcal{R}$ is not defined on the whole space $\Gamma\left(M \times \mathbb{R}^{d}\right)$. Therefore, we need to construct a suitable subspace. 
In other terms, the Plato space constructed below is also known as the set of pinpointing configurations with finite local mass, denoted by $\Gamma_{\mathrm{pf}}\left(M \times \mathbb{R}^{d}\right)$. We explore these two properties in more detail below.

Define the set of pinpointing configurations $\Gamma_{\mathrm{p}}\left(M \times \mathbb{R}^{d}\right) \subset \Gamma\left(M \times \mathbb{R}^{d}\right)$ as all configurations such that if $\left(\mu_{1}, x_{1}\right),\left(\mu_{2}, x_{2}\right) \in \gamma$ with $x_{1}=x_{2}$, then $\mu_{1}=\mu_{2}$.

Remark 5. The pinpointing property ensures that there are no two elements of a system at the same position. Due to the shape of elements in $\mathbb{K} M\left(\mathbb{R}^{d}\right)$, it is obvious that this would not be possible.

Let us now take into account the second property of $\Pi\left(M \times \mathbb{R}^{d}\right)$. To this end, we define the local mass of a configuration.

Definition 6. For a configuration $\gamma \in \Gamma_{\mathrm{p}}\left(M \times \mathbb{R}^{d}\right)$ and $\Lambda \subset \mathbb{R}^{d}$ compact, set the local mass as

$$
\gamma(\Lambda)=\int_{M \times \mathbb{R}^{d}} \mu 1_{\Lambda}(x) d \gamma(\mu, x)=\sum_{(\mu, x) \in \gamma} \mu 1_{\Lambda}(x) \in[0, \infty]
$$

This notion enables us to define the Plato space.

Definition 7. The Plato space $\Pi\left(M \times \mathbb{R}^{d}\right) \subset \Gamma\left(M \times \mathbb{R}^{d}\right)$ is defined as the space of all pinpointing configurations with finite local mass, i.e.

$\Pi\left(M \times \mathbb{R}^{d}\right):=\Gamma_{\mathrm{pf}}\left(M \times \mathbb{R}^{d}\right)=\left\{\gamma \in \Gamma_{p} \mid \gamma(\Lambda) \in \mathcal{M}\left(\mathbb{R}^{d}\right)\right.$ for all $\Lambda \subset \mathbb{R}^{d}$ compact $\}$.

Remark 8. 1) The property of finite local mass ensures that the system only has finite mass in any bounded volume, which makes it physically viable.

2) The pinpointing property as well as the finiteness of local mass are sufficient to make $\mathcal{R}: \Pi\left(M \times \mathbb{R}^{d}\right) \rightarrow \mathbb{K} M\left(\mathbb{R}^{d}\right)$ bijective.

3) The state space needs to be of the specific form $M \times \mathbb{R}^{d}$ for the notion of pinpointing configurations to make sense.

Definition 9. Let $f \in C_{c}\left(M \times \mathbb{R}^{d}\right)$ and $\eta \in \mathbb{K} M\left(\mathbb{R}^{d}\right)$. Define the following pairing:

$$
\langle\langle f, \eta\rangle\rangle:=\left\langle f, \mathcal{R}^{-1} \eta\right\rangle=\sum_{(s, x) \in \mathcal{R}^{-1} \eta} f(s, x)
$$

\section{Topology and Measure-Theoretical Structures}

In this chapter, we want to introduce a suitable topology on the cone $\mathbb{K} M\left(\mathbb{R}^{d}\right)$. To this end, we consider the topology induced on $\Pi\left(M \times \mathbb{R}^{d}\right)$ by the extended configuration space $\Gamma\left(M \times \mathbb{R}^{d}\right)$. Next, we use the mapping $\mathcal{R}$ to induce a topology on $\mathbb{K} M\left(\mathbb{R}^{d}\right)$.

\subsection{Topology on the Cone $\mathbb{K} M\left(\mathbb{R}^{d}\right)$}

The Plato space $\Pi\left(M \times \mathbb{R}^{d}\right)$ naturally inherits the topological structure of $\Gamma\left(M \times \mathbb{R}^{d}\right)$, i.e. the topology is given by the vague topology induced from the space of Radon measures $M\left(M \times \mathbb{R}^{d}\right)$. For a detailed description of topological and metric characterizations, see e.g. [4]. 
From a naive point of view, it seems to make sense to consider the embedding $\mathbb{K} M\left(\mathbb{R}^{d}\right) \subset M\left(\mathbb{R}^{d}, M\right)$ of the cone into the space of Radon measures, equipped with the vague topology. Unfortunately, this topology has no relation to the vague topology introduced above on $\Pi\left(M \times \mathbb{R}^{d}\right)$. In the spirit of Plato's theory of ideas, the connection between $\Pi\left(M \times \mathbb{R}^{d}\right)$ and $\mathbb{K} M\left(\mathbb{R}^{d}\right)$ is essential. Therefore, we consider the final topology on $\mathbb{K} M\left(\mathbb{R}^{d}\right)$ induced by the reflection mapping $\mathcal{R}$, i.e. the finest topology such that the mapping

$$
\mathcal{R}: \Pi\left(M \times \mathbb{R}^{d}\right) \rightarrow \mathbb{K} M\left(\mathbb{R}^{d}\right), \gamma=\sum_{\left(s_{x}, x\right) \in \gamma} \delta_{\left(s_{x}, x\right)} \mapsto \sum_{x \in \tau(\gamma)} s_{x} \delta_{x}
$$

is continuous. Here, we set for $\gamma \in \Pi\left(M \times \mathbb{R}^{d}\right)$,

$$
\tau(\gamma):=\left\{x \in \mathbb{R}^{d} \mid \exists s \in M:(s, x) \in \gamma\right\}
$$

the support of $\gamma$. The usage of this topology has the obvious side effect that $\mathcal{R}$ becomes a homeomorphism, which is helpful in and of itself in other regards.

In the further development of the considered theory is important to implement the construction of a class of probability measures on $\Pi\left(M \times \mathbb{R}^{d}\right)$, namely, Poisson measures. The construction may be done on the larger space $\Gamma\left(M \times \mathbb{R}^{d}\right)$. For the class of Poisson measures, we can show that they assign full mass to the Plato space $\Pi\left(M \times \mathbb{R}^{d}\right)$. To obtain measures on $\mathbb{K} M\left(\mathbb{R}^{d}\right)$, we use the pushforward of measures on $\Pi\left(M \times \mathbb{R}^{d}\right)$ under the mapping $\mathcal{R}$. A certain subclass of specific interest is the class of Gamma measures. For the detailed analysis we refer the reader to [4].

\section{Acknowledgment}

The financial support by the Ministry for Science and Education of Ukraine through Project 0119U002583 is gratefully acknowledged.

\section{References}

[1] Albeverio, S., Kondratiev, Yu.G., Röckner, M. 1998. Analysis and geometry on configuration spaces. J. Funct. Anal. 154, 444-500.

[2] Finkelshtein, D., Kondratiev, Yu.G, Lytvynov, E., Oliveira, M.J. 2022. Stirling operators in spatial combinatorics. J. Funct. Anal. 282(2), 109285.

[3] Philippe Flajolet, Robert Sedgewick. 2009. Analytic Combinatorics, Cambridge University Press.

[4] Finkelshtein, D., Kondratiev, Yu.G., Kuchling, P., Lytvynov, E., Olivera, M.J. 2021. Analysis and geometry on the cone of discrete Radon measures. Methods Funct. Anal. Topology. to appear.

[5] Kondratiev , Yu.G. 2020. Philosophy of natural numbers. Interdisciplinary studies of complex systems No.17, 34-43.

[6] Kondratiev, Yu.G., Kuna, T. 2002. Harmonic analysis on configuration space. I. General theory. Infin. Dimens. Anal. Quantum Probab. Relat. Top. 5, 201-233. 